\title{
THE REIDEMEISTER TRACE AND THE CALCULATION OF THE NIELSEN NUMBER
}

\author{
EVELYN L. HART \\ Colgate University, Department of Mathematics \\ 13 Oak Drive, Hamilton, NY 13346-1398, U.S.A. \\ E-mail: ehart@math.colgate.edu
}

1. Introduction. One goal for Nielsen fixed point theory is the capturing of geometric information in an algebraic form. The Reidemeister trace for a self-map $f$ on a finite, connected CW complex $X$ is an alternating sum of algebraic traces involving the cellular chains for the universal cover of $X$. This algebraic formal sum, when it is reduced, has for each term the index of an algebraic Nielsen class multiplied by the class itself. Thus the Nielsen number $N(f)$ is the number of terms with non-zero coefficient, and the Lefschetz number $L(f)$ is the sum of the coefficients. Wecken proved in [We, part II] that this connection between the algebraic sum and the geometric Nielsen number holds for $X$ a compact, connected polyhedron. Modern treatments of Wecken's work extend this connection to $\mathrm{CW}$ complexes (see [Hu]) and to finitely dominated spaces (see [G1]). We provide a sketch of the proof for Wecken's setting and discuss the modern treatments in Section 3. We discuss the difficulties involved in calculating the Reidemeister trace and mention some results that address these problems in Section 5. We also provide in Section 6 references for papers that include definitions of Reidemeister traces for many of the variations on Nielsen fixed point theory.

Reidemeister first defined the alternating sum of traces (see $[R]$ ) that Wecken named the Reidemeister trace ("die Reidemeistersche Spureninvariante," [We, part II]). Later the trace was also called the generalized Lefschetz number. At the conference that gave rise to these proceedings, it was generally agreed to return to the original name. Thanks are due to R. Geoghegan for helpful conversations.

Just as the Lefschetz number is defined as an alternating sum of algebraic traces and can provide information about the existence of a fixed point, the Reidemeister trace is

1991 Mathematics Subject Classification: Primary 55M20.

Key words and phrases: Reidemeister trace, generalized Lefschetz number, Nielsen number, fixed point theory.

The paper is in final form and no version of it will be published elsewhere. 
defined as an alternating sum of algebraic traces and provides information about the number of essential Nielsen classes and hence about the Nielsen number. In fact, the Reidemeister trace is zero if and only if the Nielsen number is zero.

2. The definition of the Reidemeister trace. We provide first a sketch of the necessary covering space theory. For details see [J2] or, for early sources, see [R] and [We]. We will concern ourselves here with polyhedra and discuss extensions to other spaces at the beginning of Section 3.

Let $X$ be a compact, connected polyhedron and let $f: X \rightarrow X$ be a map with $\phi: \pi \rightarrow \pi$ the homomorphism induced by $f$ on $\pi=\pi_{1}(X)$. Let $\widetilde{X}$ be the universal covering space for $X$ with $p: \widetilde{X} \rightarrow X$ the covering transformation. We consider the action of $\pi$ on $\widetilde{X}$ to be a left action, and we choose once and for all a lift of $f, \widetilde{f}: \widetilde{X} \rightarrow \widetilde{X}$ and note that every lift of $f$ can be written uniquely as $\delta^{-1} \tilde{f}$ for some $\delta \in \pi$. For any $\delta \in \pi$, we also have $\widetilde{f} \delta=\phi(\delta) \widetilde{f}$. For every $\alpha \in \pi$, the set $p \operatorname{Fix}\left(\alpha^{-1} \widetilde{f}\right)$ is either a Nielsen class of fixed points of $f$ or is the empty set. Whenever $\alpha, \beta \in \pi_{1}(X)$ with $p \operatorname{Fix}\left(\alpha^{-1} \widetilde{f}\right) \neq \emptyset$ and $p \operatorname{Fix}\left(\beta^{-1} \widetilde{f}\right) \neq \emptyset$, we have the following. The Nielsen classes $p \operatorname{Fix}\left(\alpha^{-1} \tilde{f}\right)$ and $p \operatorname{Fix}\left(\beta^{-1} \widetilde{f}\right)$ are the same if and only if there exists $\sigma \in \pi$ for which $\beta=\sigma \cdot \alpha=\phi(\sigma) \alpha \sigma^{-1}$, which means that $\alpha$ and $\beta$ are in the same orbit of the (left) Reidemeister action. Let $R(\phi)$ be the set of orbits of this action, including those that correspond to the empty set. These orbits have many names including "Reidemeister classes," "semi-conjugacy classes," "twisted conjugacy classes," and simply "algebraic classes." We have $N(f) \leq|R(\phi)| \leq\left|\pi_{1}(X)\right|$. Let $\rho: \pi \rightarrow R(\phi)$ be the function that sends $\alpha \in \pi$ to its algebraic class. Then $\rho(\alpha)$ corresponds to the set $p \operatorname{Fix}\left(\alpha^{-1} \widetilde{f}\right)$. To each algebraic class is assigned either the index of the corresponding Nielsen class or the value zero if the algebraic class corresponds to the empty set. This completes the first step in translating into an algebraic form the geometric problem of counting essential Nielsen classes. The Nielsen number equals the number of algebraic classes with non-zero index.

To incorporate the index into the algebraic statement of the problem, the Reidemeister trace of a map $f$ is defined to be a particular element of the free $\mathbb{Z}$-module $\mathbb{Z}(R(\phi))$. When this formal sum is reduced so that each algebraic class appears at most once, then the coefficient of each algebraic class equals the fixed point index of this class. Thus the Nielsen number of $f$ equals the number of terms in the reduced sum that have non-zero coefficient.

Given that $X$ is a polyhedron, we may assume without loss of generality that $f$ is simplicial because $N(f)$ is a homotopy invariant. The simplicial structure of $\widetilde{X}$ is inherited from that of $X$ so that $p: \widetilde{X} \rightarrow X$ is simplicial, and we may also assume that $\tilde{f}$ is simplicial.

The group of simplicial $q$-chains, $C_{q}(\widetilde{X} ; \mathbb{Z})$ is a free module over the group ring $\mathbb{Z}[\pi]$. The $\mathbb{Z}[\pi]$-basis consists of one lift to $\widetilde{X}$ of each $q$-cell of $X$. The Z-homomorphism induced by $\widetilde{f}, \widetilde{f}_{q}: C_{q}(\widetilde{X} ; \mathbb{Z}) \rightarrow C_{q}(\widetilde{X} ; \mathbb{Z})$, is not quite a $\mathbb{Z}[\pi]$-module homomorphism. We have for every $q$-simplex $\widetilde{u} \in C_{q}(\widetilde{X} ; \mathbb{Z})$ and for every $\sigma \in \pi, \widetilde{f}_{q}(\sigma \widetilde{u})=\phi(\sigma) \widetilde{f}_{q}(\widetilde{u})$. We define $M_{q}$ to be the matrix for $\widetilde{f}_{q}$ over $\mathbb{Z}[\pi]$, and we extend $\rho$ linearly to $\rho: \mathbb{Z}[\pi] \rightarrow \mathbb{Z}(R(\phi))$. See Section 4 for a discussion of the effect on the following definition of the choice of the lift $\tilde{f}$. 
Definition 1. The Reidemeister trace is

$$
R(f, \tilde{f}):=\sum_{q}(-1)^{q} \rho\left(\operatorname{trace} M_{q}\right) \in \mathbb{Z}(R(\phi)) .
$$

We warn the reader that some authors treat the action of $\pi$ on the cells of $\tilde{X}$ as a right action. Then we have $\widetilde{f}(\widetilde{x} \alpha)=\widetilde{f}(\widetilde{x}) \phi(\alpha)$ for $\alpha \in \pi$, and two elements $\alpha$ and $\beta$ in $\pi$ are in the same algebraic class if there is some $\sigma \in \pi$ for which $\beta=\sigma \alpha \phi\left(\sigma^{-1}\right)$.

3. The Reidemeister trace provides geometric information. We provide a sketch of Wecken's proof from [We, part II] that the Reidemeister trace, when reduced, gives both the Lefschetz number and the Nielsen number when $X$ is a compact, connected polyhedron. Modern treatments of this result are in [G1] and [Hu]. Husseini, in [Hu], extends the theorem to finite, connected CW-complexes. He also develops the theory when $H$ is a normal subgroup of $\pi$ that is invariant under $\phi$ using the corresponding intermediate covering space. This can be useful for distinguishing algebraic classes. See Section 5. Husseini then provides conditions under which the Reidemeister trace can be calculated at the level of homology. Geoghegan, in [G1], extends the following theorem to finitely dominated spaces (a connected space $X$ is finitely dominated if there is a connected finite CW complex $K$ and if there are maps $X \stackrel{u}{\rightarrow} K \stackrel{d}{\rightarrow} X$ for which $d \circ u \simeq$ $\left.\operatorname{id}_{X}\right)$. Geoghegan connects the Reidemeister trace with shape theory and algebraic $K$ theory. Specifically, he discusses connections with a $K$-theoretic conjecture of H. Bass.

TheOrem 1 (Wecken). For $X$ a compact, connected polyhedron and $f: X \rightarrow X$ a map, we have

$$
R(f, \tilde{f})=\sum_{\alpha \in W} i(\rho(\alpha)) \rho(\alpha),
$$

where $i(\rho(\alpha))$ is the fixed point index for the algebraic class $\rho(\alpha)$ and $W$ is a transversal for $R(\phi)$. Thus $N(f)$ is the number of terms of $R(f, \widetilde{f})$ with non-zero index once the sum is in this reduced form, and $L(f)$ is the sum of the coefficients.

Sketch of Proof. Using the Hopf approximation technique (see [Br] or [We, part I]), we may assume without loss of generality that $f$ is a simplicial map (from a subdivision of the original simplicial complex to the original complex) with isolated fixed points so that each fixed point is in a maximal simplex and no simplex contains two fixed points.

Once we assume that $f$ has isolated fixed points as above, the lift $\tilde{f}$ has similar properties. For each fixed point $x$ of $f$, with $x$ in the $q$-simplex $u$, there is a unique point $\widetilde{x} \in p^{-1}(x) \cap \widetilde{u}$, where $\widetilde{u}$ is the lift of $u$ that is in the $\mathbb{Z}[\pi]$-basis for $C_{q}(\widetilde{X} ; \mathbb{Z})$. There is a unique element $\alpha \in \pi$ for which $\widetilde{f}(\widetilde{x})=\alpha \widetilde{x}$. This forces the chain in $C_{q}(\widetilde{X} ; \mathbb{Z})$ that equals $\widetilde{f}_{q}(\widetilde{u})$ to contain as one of its terms either $\alpha \widetilde{u}$, if $f$ preserves the orientation of $u$, or $-\alpha \widetilde{u}$, if $f$ reverses the orientation of $u$. The contribution of $x$ to the $\mathbb{Z}[\pi]$-trace of $\widetilde{f}_{q}$ is $\pm \rho(\alpha)$ in the entry corresponding to the basis element $\widetilde{u}$. The fixed point index of $x$ is $(-1)^{q}$ if $f$ preserves the orientation of $u$ and is $(-1)^{q+1}$ if $f$ reverses the orientation of $u$. (See [G2].) Thus when the trace over $\mathbb{Z}(R(\phi))$ is multiplied by $(-1)^{q}$ in Definition 1 , the coefficient of $\rho(\alpha)$ in the diagonal entry of $\rho\left(M_{q}\right)$ corresponding to $\widetilde{u}$ becomes the fixed point index of $x$. It is certainly possible that $\rho(\alpha)$ appears again in a different diagonal 
entry of $M_{q}$. This would represent a fixed point of $f$ that was Nielsen equivalent to $x$, and thus we would have an algebraic class appearing more than once in the unreduced Reidemeister trace.

The independence of the Reidemeister trace from the various choices made (the choice of simplicial structure, the choice of the transversal $W$, etc.) must be noted. We omit the details here.

4. Properties of the Reidemeister trace. The Reidemeister trace $R(f, \widetilde{f})$ is $i n$ dependent of the choice of the lift $\tilde{f}$ of $f$ in the sense that the coefficient of $\rho(\alpha)$ in $R(f, \tilde{f})$ equals the coefficient of $\rho(\gamma \alpha)$ in $R(f, \gamma \tilde{f})$. Similarly, the Reidemeister trace is $a$ homotopy invariant. Two homotopic maps have the same Reidemeister trace if the lifts are chosen to be homotopic as well via a lift of the original homotopy. Specifically, given the homotopy $H$ from $f$ to $g$, and given the chosen lift $\widetilde{f}$, we choose the lift of $H$ to have $\widetilde{H}_{0}=\widetilde{f}$. Then we have just said that if $\widetilde{g}=\widetilde{H}_{1}$, the coefficient of $\rho(\alpha)$ is the same in the two Reidemeister traces. If the lift $\widetilde{g}$ is instead equal to $\gamma \widetilde{H}_{1}$, then the coefficient of $\rho(\alpha)$ in $R(f, \widetilde{f})$ equals the coefficient of $\rho(\gamma \alpha)$ in $R\left(g, \gamma \widetilde{H}_{1}\right)$.

The Reidemeister trace satisfies a commutativity property. Given two finite, connected CW complexes, $X$ and $Y$, and given maps $f: X \rightarrow Y$ and $g: Y \rightarrow X$, we have $R(g \circ f, \tilde{g} \circ \tilde{f})=R(f \circ g, \tilde{f} \circ \widetilde{g})$. This follows from the fact that $g(\operatorname{Fix}(f \circ g))=\operatorname{Fix}(g \circ f)$ and the fact that there is a corresponding bijection between the two sets of geometric fixed point classes.

5. The calculation of the Nielsen number. Two challenges face those who want to calculate the Nielsen number of a map by first finding the Reidemeister trace of the map. First, some workable means of finding the algebraic trace must be determined. Then the trace must be reduced so that each algebraic class appears at most once.

For $X$ a non-simply connected, closed surface and for $f$ a self-map on $X$, Fadell and Husseini prove the following in [FHu2]. Let $J(\phi)$ be the Jacobian for $\phi$ using the Fox calculus from $[\mathrm{CF}]$ and using the usual generators for $\pi$. Let $\nabla(\alpha)$ indicate the gradient in the Fox calculus for a word $\alpha$ in the free group with the same generators as $\pi$. Fadell and Husseini prove that

$$
R(f, \widetilde{f})=\rho(1-\operatorname{tr} J(\phi)+A),
$$

where $A$ is the unique element of $\mathbb{Z}[\pi]$ that satisfies $A(\nabla R)=\phi(\nabla R) J(\phi)$. An algorithm for calculating $A$ when $X$ has negative Euler characteristic is developed in [DHT], where methods for calculating $A$ for other surfaces are also discussed.

Let $X$ be a pushout space and let $f$ be a pushout map. This means that there are finite connected CW complexes $A, X_{1}, X_{2}$ so that $A$ is a subcomplex of $X_{1}$ and there are cellular maps $i_{1}: A \hookrightarrow X_{1}, i_{2}: A \rightarrow X_{2}$ so that $X:=X_{1} \bigsqcup_{i_{2}} X_{2}$. There are also cellular self-maps $f_{A}, f_{1}, f_{2}$ on the obvious spaces so that $f:=f_{1} \bigsqcup_{f_{A}} f_{2}$. In this setting, Ferrario develops in [F1] a formula for $R(f, \tilde{f})$ in terms of the Reidemeister traces of $f_{A}, f_{1}$, and $f_{2}$. This is useful for quotient spaces, union of spaces, connected sums, and wedge products.

We turn next to the problem of reducing the Reidemeister trace. Distinguishing algebraic classes is very difficult. It is related to the conjugacy problem in combinatorial 
group theory, and there is no method that is guaranteed provide an answer. We illustrate this difficulty with a simple example from [DHT], and then we list some methods for distinguishing classes that are often useful.

ExAmple 1. Let $X=T^{2} \# T^{2}$, the double torus or handcuff space, so that $\pi_{1}(X)=$ $\left\langle a, b, c, d: a b a^{-1} b^{-1} c d c^{-1} d^{-1}\right\rangle$. Let $n \geq 2$, with $f: X \rightarrow X$ chosen so that the homomorphism induced by $f$ is $\phi: \pi_{1}(X) \rightarrow \pi_{1}(X)$ given by $\phi(a)=c^{-n+1} d^{-1}, \phi(b)=d c^{n}$, $\phi(c)=a$, and $\phi(d)=b$. Using the method of Fadell and Husseini described above, we find that the Reidemeister trace is $R(f, \tilde{f})=\rho(1)+\rho\left(b a b^{-1} a^{-1}\right)$ for any $n$. But is this reduced? To determine whether the Nielsen number of $f$ is 1 or 2 , we must determine whether the group elements 1 and $b a b^{-1} a^{-1}$ are in the same orbit of the Reidemeister action (given by $\sigma \cdot \alpha=\phi(\sigma) \alpha \sigma^{-1}$ ). After [DHT] went to press, a solution to the problem was provided by Kelly. Before discussing the solution, we consider various techniques.

We return to the general situation with $X$ a finite CW complex, and we consider techniques for distinguishing algebraic classes. Given $\alpha, \beta \in \pi$ with both $\rho(\alpha)$ and $\rho(\beta)$ appearing in $R(f, \widetilde{f})$, we must determine whether $\rho(\alpha)=\rho(\beta)$. Note that we always have $\rho(\alpha)=\rho(\phi(\alpha))$, and thus $\rho(\alpha)=\rho\left(\phi^{n}(\alpha)\right)$ for all $n \in \mathbb{N}$. Thus we can calculate the images of $\alpha$ under iterates of $\phi$ to see whether any are equal to $\beta$.

If $H$ is a normal subgroup of $\pi$ for which $\phi(H) \subseteq H$, we can consider the induced action on $\bar{\pi}=\pi / H$. See [McC]. If the images $\bar{\alpha}$ and $\bar{\beta}$ in $\bar{\pi}$ are in distinct algebraic classes of the induced action of $\bar{\pi}$ on itself, then we know that $\rho(\alpha)$ and $\rho(\beta)$ are also distinct. This is particularly useful when $\bar{\pi}$ is the abelianization of $\pi$. For any abelian fundamental group, the set $R(\phi)$ corresponds to Coker $(1-\phi)$. This process is refined and developed for certain fundamental groups by Ferrario in [F2].

One method that can in some cases determine very quickly that two algebraic classes $\rho(\alpha)$ and $\rho(\beta)$ are equal is to use a computer algebra system such as Magma [BC] to calculate, for a given $\alpha \in \pi$ considered as an element of the corresponding free group, the word $\sigma \cdot \alpha$ as $\sigma$ ranges over all words of length at most $n$ for some $n \in \mathbb{N}$. The computer can compare $\sigma \cdot \alpha$ to $\beta$. See [Wa] for an algorithm that distinguishes classes for certain maps on surfaces with boundary.

At times bounds on $L(f)$ (which is the sum of the coefficients in the Reidemeister trace) can help to distinguish algebraic classes. Jiang and Guo in [JG] prove that when $f$ is a self-homeomorphism on $M$ a closed connected surface with the Euler characteristic $\chi(M)$ less than zero, then

$$
|L(f)-\chi(M)| \leq N(f)-\chi(M) .
$$

If all coefficients in the unreduced sum $R(f, \widetilde{f})$ are positive, then this inequality provides bounds on the number of terms in $R(f, \widetilde{f})$ that can be combined. This result was used in Example 4 of [HK]. In [K], Kelly extends this inequality and others to certain classes of self-maps $f$ of compact, connected surfaces with boundary for which $f$ is not homotopic to a homeomorphism. In [J3] this is extended to all maps.

Finally we return to Example 1. We must determine whether the group elements 1 and $b a b^{-1} a^{-1}$ are in the same orbit of the Reidemeister action. For this example, we did not find quotient groups helpful. The computer algebra system Magma [BC] told us 
that 1 is not $\phi$-conjugate to $b a b^{-1} a^{-1}$ using a word of length 8 or less, but even if the computer can be trusted to be correct, this does not help us. The answer, suggested by Kelly, can be found by applying the following fact from [JG]. For $X$ a compact surface with $\chi(X)<0$, and for $f$ a self-homeomorphism of $X$, let $i(\rho(\alpha))$ be the fixed point index of the algebraic class $\rho(\alpha)$. Then $2 \chi(X)-1 \leq i(\rho(\alpha)) \leq 1$. Thus in this example we have $-5 \leq i(\rho(\alpha)) \leq 1$, so that [1] cannot equal $\left[b a b^{-1} a^{-1}\right]$. So $N(f)$ is 2 . The techniques of Ferrario in $[\mathrm{F} 2]$ were also able to distinguish these two classes.

6. Other Reidemeister traces. We have omitted many theoretical results concerning the Reidemeister trace and have emphasized those that at this time are directly helpful for the calculation of the Nielsen number. Also, just as there are several types of Nielsen fixed point theories, there are several corresponding Reidemeister traces. We summarize the work done for the latter.

For relative Nielsen theory, defined by Schirmer [S] on a pair $(X, A)$ where homotopies are relative to $A$, the relative Reidemeister trace is developed by Norton-Odenthal and Wong in $[\mathrm{NW}]$. They include examples of calculations of the relative Nielsen number using the relative Reidemeister trace, and they discuss a connection with the fixed point theory of fiber preserving maps.

Local Nielsen theory, first developed in [FHu1], involves an open subset $U \subseteq X$ and a map $f: U \rightarrow X$. A local Reidemeister trace is defined in [FHa] and further developments appear in $[\mathrm{Ha}]$.

For one-parameter fixed point theory, where $F: X \times I \rightarrow X$ and $\operatorname{Fix}(F)=\{(x, t)$ : $F(x, t)=x\}$, Geoghegan and Nicas have developed a Reidemeister trace that can detect periodic orbits of flows. See [GN1] and [GN2] and also their paper that appears in this volume.

A Reidemeister trace for equivariant Nielsen fixed point theory is being developed by Wong [Wo]. In [J1], Jiang uses the mapping torus and the Reidemeister traces of iterates of a map $f$ to estimate the growth rate of the sequence of Nielsen numbers $\left\{N\left(f^{n}\right)\right\}$.

\section{References}

[BC] W. Bosma, J. J. Cannon, and G. Mathews, Programming with algebraic structures: Design of the Magma language, in: Proceedings of the 1994 International Symposium on Symbolic and Algebraic Computation, Oxford, July 20-22, 1994, M. Giesbrecht (ed.), Association for Computing Machinery, 1994, 52-57.

[Br] R. F. Brown, The Lefschetz Fixed Point Theorem, Scott, Foresman and Co., 1971.

[CF] R. Cromwell and R. Fox, An Introduction to Knot Theory, Ginn and Co., 1963.

[DHT] O. Davey, E. Hart and K. Trapp, Computation of Nielsen numbers for maps of closed surfaces, Trans. Amer. Math. Soc. 348 (1996), 3245-3266.

[FHu1] E. FADELL and S. HusSEINI, Local fixed point theory for non-simply connected manifolds, Illinois J. Math. 25 (1981), 673-699.

[FHu2] E. FADELL and S. Husseini, The Nielsen number on surfaces, in: Topological Methods in Nonlinear Functional Analysis (Toronto, Ont., 1982), Contemp. Math., 21, Amer. Math. Soc., Providence, R.I., 1983, 59-98. 
[FHa] J. FARES and E. HART, A generalized Lefschetz number for local Nielsen fixed point theory, Topology Appl. 59 (1994), 1-23.

[F1] D. Ferrario, Generalized Lefschetz numbers of pushout maps, Topology Appl. 68 (1996), 67-81.

[F2] D. Ferrario, Computing Reidemeister classes, Fund. Math. 158 (1998), 1-18.

[G1] R. Geoghegan, Fixed points in finitely dominated compacta: the geometric meaning of a conjecture of H. Bass, in: Shape Theory and Geometric Topology (Dubrovnik 1981), S. Mardešić and J. Segal (eds.), Lecture Notes in Math., 870, Springer-Verlag, 1981, 6-22.

[G2] R. Geoghegan, Nielsen Fixed Point Theory, in: Handbook of Geometric Topology, R. J. Daverman and R. B. Sher (eds.), to be published by Elsevier.

[GN1] R. Geoghegan and A. NiCAs, Parametrized Lefschetz-Nielsen fixed point theory and Hochschild homology traces, Amer. J. Math. 116 (1994), 397-446.

[GN2] R. Geoghegan and A. Nicas, Trace and torsion in the theory of flows, Topology 33 (1994), 683-719.

[Ha] E. HART, Computation of the local generalized H-Lefschetz number, Topology Appl. 61 (1995), 115-135.

[HK] E. HART and E. KePpelmann, Explorations in Nielsen periodic point theory for the double torus, Topology Appl. 95 (1999), 1-30.

[Hu] S. Husseini, Generalized Lefschetz numbers, Trans. Amer. Math. Soc. 272 (1982), 247-274.

[J1] B. JIANG, Estimation of the number of periodic orbits, Pacific J. Math. 172 (1996), 151-185.

[J2] B. JiAng, Lectures on Nielsen Fixed Point Theory, Contemp. Math. 14, Amer. Math. Soc., Providence, Rhode Island, 1983.

[J3] B. JiAnG, Bounds for fixed points on surfaces, Math. Ann. 311 (1998), 467-479.

[JG] B. Jiang and J. Guo, Fixed points of surface diffeomorphisms, Pacific J. Math. 160 (1993), 67-89.

[K] M. KeLLY, Minimal surface maps, fixed point indices and a Jiang/Guo type inequality, these proceedings.

[McC] D. McCord, An estimate of the Nielsen number and an example concerning the Lefschetz fixed point theorem, Pacific J. Math. 66 (1976), 195-203.

[NW] B. Norton-Odenthal and P. Wong, A relative generalized Lefschetz number, Topology Appl. 56 (1994), 141-157.

[R] K. Reidemeister, Complexes and homotopy chains, Bull. Amer. Math. Soc. 56 (1950), 297-307.

[S] H. Schirmer, A relative Nielsen number, Pacific J. Math. 122 (1986), 459-473.

[We] F. Wecken, Fixpunktklassen, I, II, III, Math. Ann. 117 (1941), 659-671; 118 (1942), 216-234 and 544-577.

[Wa] J. WAGNER, An algorithm for calculating the Nielsen number on surfaces with boundary, Trans. Amer. Math. Soc. 351 (1999), 41-62.

[Wo] P. Wong, private communication, 1996. 